\title{
Structure-function analysis of Xanthomonas oryzae pv. oryzae virulence factor CbsA
}

$\underline{\text { Rajkanwar Nathawat }}{ }^{1}$, Tayi Lavanya ${ }^{1}$, sushil Kumar ${ }^{2}$, Ramesh V Sonti ${ }^{1}$, Rajan Sankaranarayanan ${ }^{1}$

${ }^{1}$ Center For Cellular \& Molecular Biology, Hyderabad, India, ${ }^{2}$ Institute of Life Sciences, Bhubaneswar, India E-mail: rajkanwar@ccmb.res.in

Xanthomonas oryzae pv. oryzae (Xoo) causes bacterial blight disease of rice. Xoo uses cell wall-degrading enzymes like Cellulase, Esterase (LipA), Cellobiosidase, Xylanase, etc. as virulence factors. As part of our on-going efforts in understanding the structural basis of these virulence factors, structure of LipA and CbsA (Catalytic domain) proteins were solved. LipA protein belongs to a new class of cell wall-degrading enzymes which has a unique mode of substrate recognition. CbsA protein has an $\mathrm{N}$-terminal catalytic domain (Glycosyl hydrolase family-6) and a C-terminal fibronectin type 3 domain. Under laboratory conditions, Xoo-secreted CbsA is a truncated protein having just the catalytic domain. The structure reveals the presence of the catalytic tunnel being enclosed by two loops, characteristic of a typical exoglucanase. Based on the structure, key residues important for catalysis were predicted. These residues are D99, S105, D148, Y93 and D367. In Glycoside hydrolase family 6 there is general agreement as to the identity of the catalytic acid from both crystallographic and solution studies. In case of CbsA this residue is D148. Surprisingly, CbsA D148A mutant shows catalytic activity. To determine if the enzymatic activity is required for its ability to induce immune responses and for its role in virulence, biochemically inactive forms of CbsA which abrogate the enzymatic activity are being generated.

The C-terminal region of CbsA is the fibronectin type 3 (FN3) domain. FN3 domain has been reported in bacterial carbohydrate active enzymes, where it is implicated in binding of sugar groups or promotes hydrolysis. However, the distinctive feature of carbohydrate binding module, such as a large aromatic platform, is not detected on the FN3 domain. Cell fractionation studies indicate that the FN3 domain of CbsA is cleaved off in the cytoplasm. CbsA protein without the FN3 domain is biochemically active and can induce immune response in rice tissues. To assess the role of the fibronectin domain in the function of CbsA, a deletion mutant of FN3 domain was generated in Xoo and this mutant was found to be deficient in virulence. Western blot analysis using anti-CbsA antibody indicated that the level of the secreted CbsA protein in the $\triangle \mathrm{FN} 3$ mutant was around $95 \%$ less than the wild type. However, RT-PCR analysis showed equal levels of cbsA gene expression in $\triangle$ FN3 mutant and wild type. Taken together, these results suggest that the deletion of the fibronectin domain results in reduced level of secreted CbsA protein, either due to altered post-transcriptional processing or transport across the membrane.

1. Aparna G. et al. (2009) The Plant Cell. 21: 1860-1873

2. Jha et al. (2007) Mol. Plant Microbe Interact. 20: 31-40

3. Kumar S. et al. (2012) Acta Cryst. F68, 1191-1194

Keywords: virulence, exoglucanase, immune 